\title{
Manuscript Reviewers
}

The following individuals generously offered their time and expertise as manuscript reviewers on one or more occasions between 11 December 2019 and 20 December 2020. We are grateful for their assistance.

\begin{tabular}{|c|c|c|c|}
\hline Ackrén, M. & Dowsley, M. & Leppänen, J. & Robards, M. \\
\hline Alder, S. & Duerden, F. & Leseto, L. & Roland, C.A. \\
\hline Anderson, D. & Durner, G.M. & Linderholm, A.E. & Rolph, R. \\
\hline Anderson, K. & Elliot, G.P. & Ljubicic, G. & Rozenfeld, S. \\
\hline Archambault, P. & Fediuk, K. & Lowan-Trudeau, G. & Saku, J.C. \\
\hline Asgary, A. & Ferguson, S. & Lundgren, R. & Saxinger, G. \\
\hline Bakaic, M. & Gallagher, C. & Lunn, N. & Schmidt, J.H. \\
\hline Barbeau, C. & Galloway, T. & Mallory, C. & Schmutz, J.A. \\
\hline Barnhart, T. & Gaston, A. & McTavish, K. & Schott, S. \\
\hline Beatty, B. & Gaudry, A. & Miller, J.A. & Shea, J. \\
\hline Behe, C. & Goldhar, C.A. & Moore, S. & Sidorova, J. \\
\hline Bernauer, W. & Grimes, V. & Nadasdy, P. & Slocombe, S.D. \\
\hline Biskaborn, B.K. & Gunn, A. & Natcher, D. & Smith, E.A. \\
\hline Boertmann, D. & Gunn, J. & Nawrocki, T. & Smith, H. \\
\hline Bogdan, A.-M. & Gunster, S. & Nilsson, L.M. & Smol, J. \\
\hline Boveng, P. & Halliday, W. & Noble, B. & Smultea, M. \\
\hline Brook, R. & Hamel, S. & Nobmann, E.D. & Snow, K. \\
\hline Bulla, M. & Harwood, L. & North, M.R. & Sohns, A. \\
\hline Burn, C. & Haskell, S & Nurmi, R. & Spring, A. \\
\hline Calverley, D. & Hellström, P. & Obbard, M. & Starblanket, G. \\
\hline Carayannis, E. & Henri, D. & Ogden, A. & Stecyk, K. \\
\hline Chetkiewicz, C. & Herman-Mercer, N. & Ostertag, S. & Stern, H. \\
\hline Cidro, J. & Hodgson, E. & Pantzer, B. & Stewart, E. \\
\hline Clark, D. & Huntington, $\mathrm{H}$. & Parker, C. & Stirling, I. \\
\hline Coates, $\mathrm{K}$. & Hupp, J.W. & Paszlowski, C. & Taylor, $\mathrm{M}$. \\
\hline Coleman, J. & Johnson, C.-A. & Pearce, T. & Têtu, P.-L. \\
\hline Cost, D. & Kao, S.-M. & Pilaar Birch, S.E. & Thiemann, G. \\
\hline Crawford, J. & Karlsson, M. & Pinus, R. & Titz, A. \\
\hline Cuyler, C. & Katz, J. & Plummer, R. & Tyler, N. \\
\hline Dabros, A. & Kelly, B. & Pollock, N. & Vaguet, V. \\
\hline Daniels, B. & Koutouki, K. & Pretty, J. & Vayro, J. \\
\hline Dankel, D.J. & Kruemmel, E. & Putkonen, J. & Veland, S. \\
\hline Dariment, $\mathrm{C}$. & Kullman, L. & Rachold, V. & Vonk, J. \\
\hline Datta, R. & Kuo, C.-C. & Ramage, J. & West, C.T. \\
\hline Davis, K.M. & Kutz, S. & Raymond, J. & Wilson, C. \\
\hline Dearborn, K. & Lackenbauer, P.W. & Reed, M. & Wilson, N. \\
\hline Derocher, A. & Lajeunesse, A. & Reinfort, B. & Wilson, R. \\
\hline Donovan, S. & Leblond, M. & Resouli, K. & Wiseman, D. \\
\hline Douglas, M. & Lehtinen, K. & Richardson, E. & $\begin{array}{l}\text { Wright, N. } \\
\text { Zitterbart, D.P. }\end{array}$ \\
\hline
\end{tabular}

